\title{
Kernos
}

Revue internationale et pluridisciplinaire de religion grecque antique

18 | 2005

Varia

\section{Hommage à Pierre Lévêque}

Pierre Brulé

\section{OpenEdition}

Journals

Édition électronique

URL : http://journals.openedition.org/kernos/871

DOI : $10.4000 /$ kernos.871

ISSN : 2034-7871

\section{Éditeur}

Centre international d'étude de la religion grecque antique

Édition imprimée

Date de publication : 1 janvier 2005

Pagination : 11-15

ISSN : 0776-3824

Référence électronique

Pierre Brulé, « Hommage à Pierre Lévêque », Kernos [En ligne], 18 | 2005, mis en ligne le 05 mai 2011, consulté le 01 mai 2019. URL : http://journals.openedition.org/kernos/871 


\section{Hommage à Pierre Lévêque}

Pierre Lévêque est mort le 5 mars 2004 à 82 ans.

Il avait toujours surpris par une activité débordante qui le portait aux quatre coins du monde, franchissant avec une aisance déconcertante latitudes et méridiens. Robert Étienne lui disait : "Notre oikoumène suffit à peine à borner tes errances. » Il en allait de même avec siècles et millénaires et les terrains d'enquête. «On me reproche, » m’écrit-il un jour, «mon mysticisme de la recherche. » Mysticisme ? Je ne sais pas. Mais, quelle gourmandise ! Pour ne parler que d'histoire ancienne, quelle variété d'intérêts il a toujours montrée, tirant jubilation intellectuelle aussi bien des dévaluations à Rome que de la sculpture - qu'elle vienne de Délos, de Thasos, de Delphes, de Rhodes ou du Portugal... -, du commerce de l'étain que des poètes alexandrins, des Hittites que des Doriens. Il vibrait d'une libido sciendi qui le faisait embrasser toujours de nouveaux projets, lancer de nouvelles enquêtes, accueillir de nouveaux chercheurs. Son goût pour les périples le conduisait en Géorgie, en Grande Grèce, mais aussi au Brésil, au Canada et au Japon. Il n'était pas l'homme des petits cantons - son Érétrie était «Rameuse»-, son regard périphérique (là où une aventure est toujours en réserve) l'attirait vers des marges, de la Gaule aux royaumes gréco-bactriens et gréco-indiens; ainsi s'explique sans doute aussi son intérêt toujours vif pour les phénomènes de colonisation - encore le voyage. Le voyage, c'est cette autre appétence pour la pensée des autres. Ainsi, à partir de la fin des années 1970, vient s'ajouter cette autre invitation à l'aventure, celle qui lui a fait saisir l'Antiquité classique à travers le miroir des expériences d'autres sociétés ${ }^{1}$. Et, au sein de la culture grecque elle-même, c'est avec Pierre Vidal-Naquet qu'il mit au point une telle image réfractée, donnant un grand coup de jeune à des Clisthène et Épaminondas.

Forts de notre décalage chronologique, nous voyons dans ses années de formation et dans l'entrée de Pierre Lévêque dans le concert des historiens de l'Antiquité le clair présage de ces développements tous azimuts : cet éclec-

\footnotetext{
1 Voir deux articles de la riche décennie 1975-1985: «Religions africaines et religion grecque : pour une analyse comparée des idéologies religieuses », Afrique noire et monde méditerranéen dans l'Antiquité, Colloque de Dakar, 19-24 janvier 1976, Dakar-Abidjan, 1976, p. 196236 [repris dans La Pensée, 210 (févr. 1980), p. 30-62] et "Approche ethno-historique des concours grecs », Klio 64, 1 (1982), p. 5-20 [Mélanges H. Kreissig] (où la comparaison est menée avec les Mayas).
} 
tisme, qui était aussi un cosmopolitisme, cette curiosité toujours prête à se saisir d'un nouvel objet. On qualifierait aujourd'hui ses trois premiers ouvrages de «décalés », par leur sujet, et par ce qu'ils supposaient d'emblée d'une connaissance multiforme des sociétés antiques - il fallait avoir acquis pour cela aussi bien la maîtrise de la sculpture archaïque que celle du théâtre classique et des néo-platoniciens ${ }^{2}$. Or il se trouve que l'on ne prise pas trop l'éclectisme en ce milieu... Le choix de Pyrrhos comme sujet de thèse est symptomatique, comme il est unique, en ce temps comme aujourd'hui encore. Il cumule les originalités. Une biographie. Un souverain d'une Grèce très mal connue. Un destin mêlant deux mondes. Tout est décalage. Comme l'est son Agathon et plus encore son Aurea catena Homeri : une étude sur l'allégorie grecque ${ }^{3}$. On imagine le foisonnement des lectures en tous sens. Et, dans la continuité du chercheur multivalent, est vite venu ce livre, nourri en outre d'une inhabituelle sympathie avec son sujet, et que tant d'historiens - fort nombreux en dehors des spécialistes - considèrent comme un chef-d'œuvre de synthèse, son Aventure grecque ( $1^{\mathrm{e}}$ éd., 1964, 6 éditions et 5 traductions). Nos milieux rechignent aux hyperboles, mais, pour évoquer ce livre, il faudrait pouvoir restituer l'enthousiasme qu'il a suscité. Qui a écrit des manuels, ou, plus exactement, de telles vastes synthèses, sait à quel point il y a loin d'un «état des questions » à ce véritable poème. Nonobstant les très grandes qualités d'historien d'un André Aymard (plus grandes qualités sans doute), mesurez la distance qui sépare de ce point de vue L'Orient et la Grèce... de L'aventure. En aval, on soutiendra aussi qu'en retour, les milliers de fiches nécessaires à L'aventure expliquent cette aisance évoquée plus haut à disserter des sujets les plus divers. Mais ce serait, je crois, trahir la démarche de Pierre Lévêque que de négliger les motivations impérieuses qui suscitaient les réponses d'un humaniste traditionnel. Ces motivations, c'était le choc de la pensée des autres. C'est ainsi qu'on le voit, successivement ou synchroniquement, abonder dans le dumézilisme, le structuralisme, le marxisme, - sans parler de son intérêt pour les procédés optiques de déchiffrage des cadastres... Souplesse impressionnante de la pensée, à l'affût des «derniers » renseignements sur tel ou tel sujet (pour prendre un exemple : sa passion subite pour les amphores SOS). Autant d'outils, autant de tremplins pour penser.

«Dans le fond, il n’y a que cela qui m'intéresse...» C'est de religion grecque qu'il me parlait. Je ne sais pas si cette parole est venue tard dans son parcours intellectuel (je l'ai entendue en 1988). Toutefois, dans sa vaste bibliographie, on peut trouver les antécédents anciens de cet aveu. Sans compter

\footnotetext{
${ }^{2}$ Le seul point aveugle (relatif) de Pierre Lévêque, c'est l'épigraphie - il y a là la trace d'une hiérarchie des valeurs préjudiciables à une juste prise en compte des rituels dans ses travaux sur la religion.

3 Respectivement : Paris, 1955 et Paris 1959; Pyrrbos, Paris, 1957, 735 p.

4 Qui fut un de ses maîtres.

A. Aymard et J. Auboyer, L'Orient et la Grèce antique, Paris, PUF, 1953
} 
les pages que L'aventure lui consacre, le premier signe de cet intérêt, c'est évidemment ce maître-livre aussi que constitue Les grandes divinités de la Grèce $e^{6}$, entrepris en collaboration avec Louis Séchan, cet helléniste qui l'avait lancé sur la piste d'Agathon ${ }^{7}$. Dix ans après sa sortie, il regrettera néanmoins en raison des compromis impliqués par une telle collaboration - d'avoir dû modérer son désir d'une présentation moins «traditionnelle » des dieux grecs. Quoi qu'il en soit, le projet de l'ouvrage - tout dire sur chaque dieu interdisait évidemment la formulation de perspectives synthétiques.

À qui cherche dans l'Index des Mélanges Pierre Lévêque (M.-M. Mactoux et E. Gény [éds] 1995) la trace de la première formulation de ce qui caractérisera la pensée de Pierre Lévêque sur la religion grecque, il faut attendre longtemps. Le premier ouvrage synthétique qu'il lui consacre date de 1985, c'est Bêtes, dieux et hommes. L'imaginaire des premières religions. Ce livre-bilan avait été préparé par une longue suite d'études où avaient été mises à l'épreuve les principales idées qui y sont formulées. Mais ce socle lui-même, à la fois l'idée que Pierre Lévêque se faisait de sa recherche et aussi le noyau de sa conception, se repère plus tôt encore dans un texte malheureusement non publié. C'est en 1968, l'année où fut mise au programme des concours de recrutement de l'enseignement la question sur la religion grecque ${ }^{9}$. Dans ce cours ronéotypé rédigé pour les étudiants de Besançon, et qui va du néolithique au Corpus hermétique, en passant par l'iconographie minoenne, les tablettes mycéniennes, l'architecture religieuse, l'origine du théâtre, Pierre Lévêque fournit, me semble-t-il, les premières réponses aux questions qu'il pose à l'histoire de cette religion. On y voit déjà les essais de reconstitution des panthéons protohistoriques, y sont agitées les questions de la continuité des cultes sur la longue durée, des syncrétismes, des Doriens, des hiérogamies, de l'eschatologie dans ses liens avec le culte des forces de vie, de la signification religieuse des concours. L'auteur tente de répondre dans la diachronie et, que ce soit par le recours à l'archéologie (toujours sollicitée comme pièce à conviction) ou à l'étymologie, pointe constamment ce souci d'une quête de l'origine des dieux, moins des rites et un peu des mythes. Déjà à cette époque, il milite pour une continuité globale depuis le II millénaire et ne voit de chiasme qu'à l'époque historique, vers 430, quand "l'inquiétude étreint les consciences» (p. 154 du vol. 2). Ainsi, tout ce qui constituera la matière des recherches ultérieures de Pierre Lévêque est présent dès 1968 (c'est l'année de la fondation du Centre de Besançon).

Viennent les années 1970 avec cette superbe et fort cohérente série d'articles : «Formes et structures méditerranéennes dans la genèse de la reli-

${ }^{6}$ Paris, de Boccard, 1966 (2 éd. augmentée, A. Colin, 1990).

7 Et l'avait aussi entraîné jusqu'à passer ses dimanches à réviser notre Bailly.

8 Paris, Messidor, 240 p.

9 La chose n'était évidemment pas absolument nouvelle, et un article comme celui sur les cultes d'Arcadie, de 1961, apparaît en quelque sorte prémonitoire : "Sur quelques cultes d'Arcadie: Princesses-ourses, hommes-loups et dieux-chevaux », L'Information historique 23 (1961), p. 93-108. 
gion grecque », "Continuités et innovations dans la religion grecque dans le première moitié du I ${ }^{\mathrm{er}}$ millénaire », «Essai de typologie des syncrétismes », et "Le syncrétisme créto-mycénien » qui clôt ce cycle. Trop d'obligations ne lui laissent qu'un temps limité pour écrire sur ce qui «[l]'intéresse vraiment». Pourtant, son extraordinaire facilité aidant, cela ne l'empêche pas d'écrire beaucoup. Peut-on dire trop ? Quoi qu'il en soit, Pierre Lévêque ne reprendra plus ce dossier d'une sorte d'archéologie de la religion grecque avant 1981.

La seconde volée des articles qui vont le mener aux Bêtes..., opère un virage dans son approche. Désormais plus soucieux d'articuler pensée marxiste et histoire, plus influencé alors par les lectures de Jeanmaire, de Gernet et de Brelich, mais toujours mu par le goût du voyage vers les origines, le comparatisme entre par la grande porte pour deux types de relecture : d'une part celle du plus lointain passé : «À propos des chasseurs du Nil et du Sahara : la pensée des chasseurs archaïques » et "Contribution à une théorie historique de la production de la pensée religieuse dans les sociétés du Paléolithique et du Néolithique »" ${ }^{11}$, et d'autre part celle de questions spécifiques de la religion grecque comme les concours et les mystères : «Approche ethno-historique des concours grecs », «Concours grecs, jeux romains et jeux mésoaméricains. L'idéologie de l'éternel retour »" «Olbios et la félicité des initiés » et «Structures imaginaires et fonctionnement des mystères grecs ». À lire les Bêtes dans la continuité de cette double volée de travaux, il apparaît clairement que toute l'œuvre a tendu vers l'écriture de ce livre, son vrai projet.

«Ce qui importe, à mon sens, c'est de développer une étude résolument historique $^{14}$ de la religion grecque, c'est-à-dire de tenter de saisir la genèse des mythes et des rites, d'appréhender les relations de causalité entre les infrastructures socio-économiques et les faits religieux, de suivre et d'expliquer l'évolution sous la double forme des permanences et des innovations ${ }^{15}$. Cela fut longtemps sa doctrine. Cela s'applique, par exemple, à l'enfant divin. Pierre Lévêque accumule les traits de ce qu'il considère comme une structure : naissance et enfance " anormales », mise en danger, rapports aux "nymphes » bienveillantes, passage par les Enfers, hieros gamos avec une Grande-

10 Respectivement : Praelectionnes Patavinae, Rome, 1972, p. 145-180; PP 28 (1973), p. $23-$ 50; Les syncrétismes dans les religions grecques et romaines, Actes du colloque de Strasbourg, 9-11 juin 1971, Paris, PUF, 1973, p. 179-87; Les syncrétismes dans les religions de l'Antiquité, sous la direction de Fr. Dunand et P. Lévêque, Colloque de Besançon, 22-23 oct. 1973, Leiden, Brill, 1975 , p. $19-75$

Deux articles du DHA 7 (1981), p. 41-52 et 53-92

12 Klio 64 (1982), p. 5-20; Archaiologia, Athènes, 4 (1982), p. 8-16.

13 Rayonnement grec. Hommages à Charles Delvoye, Bruxelles, 1982, p. 113-126; SSR 6 (1982), p. 185-208.

Les italiques sont d'origine.

15 «Un nouveau décryptage des mythes d'Adonis », REA 74 (1972), p. 180-185, texte polémique rendant compte du livre de M. DETIENNE, Les jardins d'Adonis. La mythologie des aromates en Grèce (1972). Il est piquant d'y lire aujourd'hui ce reproche à l'auteur, d'avoir condamné un « comparatisme sans frein » (p. 183). 
Mère, connexions avec les fleurs, mort lamentable, résurrection annuelle (Pierre Lévêque avoue ce qu'il doit à Frazer et aussi, un peu, à M.P. Nilsson, à Ch. Picard, moins à J. Harrison). Ce qui caractérise la procédure intellectuelle de Pierre Lévêque, c'est qu'il considère avoir fait la preuve de la validité de ce modèle structural par une induction dans le passé. S'il est possible d'en reconstituer la genèse, la preuve sera faite de la réalité du modèle. D'où cette quête qui le mène aux Mycéniens, aux Minoens, au néolithique de Çatal Hüyük, et plus tard, en route vers les Bêtes..., jusqu'au paléolithique. C'est-àdire que, d'une invariance définie pour une époque donnée, il cherche à reconstituer une évolution, mais il se révèle que c'est une «téléonomie ». Le paradoxe de sa méthode c'est que ce qu'on retiendra de meilleur, de plus salutaire, à savoir son souci d'histoire, l'ait plus amené vers un « comparatisme chronologique » que vers une histoire véritable, ployant à outrance les réalités historiques. Prenons une Artémis. L'oubli de sa réalité à l'instant $t$ suppose de la faire entrer dans la diachronie à toute épreuve d'un concept aussi flou que celui de la Grande Mère néolithique, déesse de fertilité et de fécondité, - elle qui ne connut jamais de ces « embrassements», ni cosmiques ni autres.

Écrire un hommage, c'est manifester une fidélité. Mais, par incompétence, je laisse à d'autres d'autres regards, et je finirai par le «noyau dur» : une gratitude pour le plaisir de cette ouvre immense et passionnante qui nous a nourris, pour nous avoir donné le goût, la foi en l'histoire, enfin, à une époque où cela n'allait pas de soi, pour avoir rendu possible l'expression des jeunes chercheurs. Quant à ce qui m'intéresse ici, l'histoire de la religion grecque, elle a beaucoup à gagner à l'application du programme de Pierre Lévêque. Mais une histoire consciente de ses limites documentaires et attentive aux pièges des raisonnements inductifs où la découverte est trop souvent, déjà, dans la question.

Pierre BRULÉ 